\title{
Stability of PS Opals in Supercritical Carbon Dioxide and Synthesis of Silica Inverse Opals
}

\author{
Hye Min Yu, Ah Ram Kim, Jun Hyuk Moon, Jong Sung Lim, and Kyu Yong Choi ${ }^{\dagger}$ \\ Department of Chemical and Biomolecular Engineering, Sogang University, Seoul 121-742, Korea. *E-mail: limjs@sogang.ac.kr \\ ${ }^{\dagger}$ Department of Chemical and Biomolecular Engineering, University of Maryland, MD 20742, USA \\ Received October 19, 2011, Accepted May 9, 2011
}

\begin{abstract}
Recently, the synthesis of ordered macroporous materials has received much attention due to its potential use as photonic band gap materials. ${ }^{1}$ In this study, we have used the three-dimensional (3D) latex array template impregnated with benzenesulfonic acid (BSA), which is capable of catalyzing the reaction using tetraethyl orthosilicate (TEOS) as a precursor and distilled water. The polystyrene (PS) templates were reacted with TEOS in $\mathrm{scCO}_{2}$ at $40{ }^{\circ} \mathrm{C}$ and at 80 bar. In the reactor, TEOS was filtrated into the PS particle lattice. After the reaction, porous silica materials were obtained by calcinations of the template. The stability test of the PS template in pure $\mathrm{CO}_{2}$ was conducted before the main experiment. Scanning electron microscopy (SEM) images showed that the reaction in $\mathrm{scCO}_{2}$ takes place only on the particle surface. This new method using $\mathrm{scCO}_{2}$ has advantages over conventional sol-gel processes in its capability to control the fluid properties such as viscosity and interfacial tension. It has been found that the reaction in $\mathrm{scCO}_{2}$ occurs only on the particle surface, making the proposed technique as more rapid and sustainable method of synthesizing inverse opal materials than conventional coating processes in the liquid phase and in the vapor phase.
\end{abstract}

Key Words : Supercritical carbon dioxide, Silica inverse opals, TEOS

\section{Introduction}

Three-dimensional (3D) photonic crystals (PCs) are crystalline materials in which the refractive index is periodically modulated on a length scale that is comparable to the light wavelength of interest. ${ }^{2}$ These materials share the unique photonic band gap (PBG), which prohibits the propagation of a particular range of electromagnetic frequencies through the structure. ${ }^{3}$ Therefore, it is possible to tailor the emissive properties of internal light sources by controlling the PBG. To have a complete PBG, the structure must be extremely porous with a specific sub-micrometer $3 \mathrm{D}$ periodicity, and the materials must have a high refractive index and negligible absorption at the desired wavelengths. ${ }^{4}$ There have been many approaches to fabricate 3D PCs to satisfy this requirement. ${ }^{5,6}$ Of these, colloidal crystals have been found to be one of the simplest and fastest chemical assembly methods.

Colloidal crystals are regular crystalline arrays of highly monodisperse colloids of dielectric materials, such as silica or polymer. One natural example is the gemstone opal. ${ }^{7}$ However, natural opals are expensive and have a characteristic in which different frequencies of light are reflected against the incident light with a specific direction. Colloidal crystals provide an ideal scaffold for the creation of a more interesting photonic crystal-inverted opal, ${ }^{8}$ a material with complex porous structures. Inverse opals have an inverted structure that is obtained by filling the voids of the opal structure with materials of high refractive index and then removing the original opal materials. Thus, inverse opals consist of a regular arrangement of spherical void spaces surrounded by solid walls rather than a regular arrangement of uniform spherical particles. Because of this special structure, inverse opals, as with porous ceramic materials, can be used in a variety of applications, including photonic crystal devices, catalysts, sorbents, chromatographic materials, biomaterials, and microelectronics. ${ }^{8}$

Since the late 1990 's, a variety of methods ${ }^{9}$ have been designed to synthesize inverse opals. Of those methods, we chose supercritical deposition. In supercritical deposition, the precursor is deposited onto the substrates using supercritical-state fluids. Supercritical fluids have attracted much attention in the synthesis of ceramic materials. If a supercritical fluid is used as a reaction medium, the interfacial tension of the reactants and the fluid density are more readily controlled because the high-density liquid state is uniform and can be continuously changed. Specifically, supercritical carbon dioxide $\left(\mathrm{scCO}_{2}\right)$ is most frequently used as a medium because of its cost effectiveness, nontoxic characteristics, and pressure requirements $\left(\mathrm{T}_{\mathrm{c}}=31{ }^{\circ} \mathrm{C}, \mathrm{P}_{\mathrm{c}}=73.8 \text { bar }\right)^{2,10}$ Compared to liquid-phase deposition, $\mathrm{scCO}_{2}$ possesses low viscosity, high diffusivity, and very low surface tension; therefore, the infiltration of precursors can be improved. ${ }^{2,11}$ In particular, 3D materials are coated, and the reactants can diffuse deep inside the particle easily.

In this paper, we investigate the fabrication of $\mathrm{SiO}_{2}$ inverse opals using $\mathrm{scCO}_{2}$. For this purpose, a 3D latex template infiltrated with tetraethyl orthosilicate (TEOS) was used. We conducted the experiments under different conditions, varying temperature, pressure, and reaction time. Sample characteristics were evaluated using scanning electron microscopy. 


\section{Experimental Section}

Materials. We used two different diameter of PS $(1.1 \mu \mathrm{m}$ and $235 \mathrm{~nm}$ ). The PS of $1.1 \mu \mathrm{m}$ diameter was purchased from Aldrich. For $235 \mathrm{~nm}$ diameter PS, it was synthesized by using emulsion free polymerization. ${ }^{12}$ For the emulsion free polymerization, distilled water, monodisperse polystyrene (Aldrich, 99\%), potassium persulfate (Kanto, 98\%), methacrylic acid (Aldrich, 99\%), and acrlic acid (Aldrich, 99\%) were used. For the silica coating on PS opal templates, the PS opals were treated by benzenesulfonic acid (Aldrich, $90 \%$ ) and, then, the silica coating of PS opals were obtained by using tetraorthosilicate (TEOS, Aldrich, 90\%) and $\mathrm{CO}_{2}$ gas.

Preparation of the PS Opal Template and the Stability Test in $\mathbf{s c C O}_{2}$. To fabricate PS opal templates, the PS colloids were dropped on a silicon wafer or glass substrate and then, dried in air. During the drying process, PS particles were assembled into PS opals. Then, each of the PS templates was treated in the reactor with pure $\mathrm{CO}_{2}$ at different temperatures and pressures. The $\mathrm{scCO}_{2}$ temperature and pressure ranges were 40 to $70{ }^{\circ} \mathrm{C}$ and 80 to $140 \mathrm{bar}$, respectively. The treatment time was varied from $30 \mathrm{~min}$ to $5 \mathrm{hrs}$.

Coating of $\mathrm{SiO}_{2}$ in $\mathbf{s c C O}_{2}$. The treated PS template was placed in a glass vial, which was loaded in a $157-\mathrm{mL}$ stainless steel high-pressure reactor in an air bath. In addition, glass vials, each containing TEOS and $\mathrm{H}_{2} \mathrm{O}$, were also loaded in this reactor. The thermostatic reactor cell was filled with $\mathrm{CO}_{2}$ using a high-pressure syringe pump until the desired experimental pressure was reached at a temperature of $40{ }^{\circ} \mathrm{C}$.

The reaction time was varied from 20 to $40 \mathrm{~min}$ in the reactor. After the reaction, $\mathrm{CO}_{2}$ gas in the reactor was slowly ventilated through a needle valve. When the reactor was depressurized, the non-reacted TEOS and other reaction byproducts, such as ethanol dissolved in the supercritical fluid, were vented from the reactor. No additional washing or extraction steps were needed. After the coating process, the samples were calcined at $500{ }^{\circ} \mathrm{C}$ for $1 \mathrm{hr}$ to remove the PS template.

Characterizations. FE-SEM (Field Emission Scanning Electron Microscope, HITACHI S-4800) has been used to investigate PS templates and silica inverse opals. Before the SEM imaging, samples were coated with Pt in vacuum for enhancing conductivity.

\section{Results and Discussion}

Stability Test of PS Template in Pure $\mathrm{ScCO}_{2}$. It has been reported that the glass transition temperature, $\mathrm{T}_{\mathrm{g}}$ of polymers can be decreased under high pressures. ${ }^{13-16} \mathrm{~T}_{\mathrm{g}}$ is the temperature above which polymers and inorganic glasses no longer behave as brittle materials. To achieve a supercritical condition for $\mathrm{CO}_{2}$, the pressure and temperature should be higher than 73.8 bar and $31^{\circ} \mathrm{C}$, respectively. Therefore, we might expect to observe decrease in the $\mathrm{T}_{\mathrm{g}}$ of PS opal templates. At temperature above $T_{g}$, annealing of the PS templates can
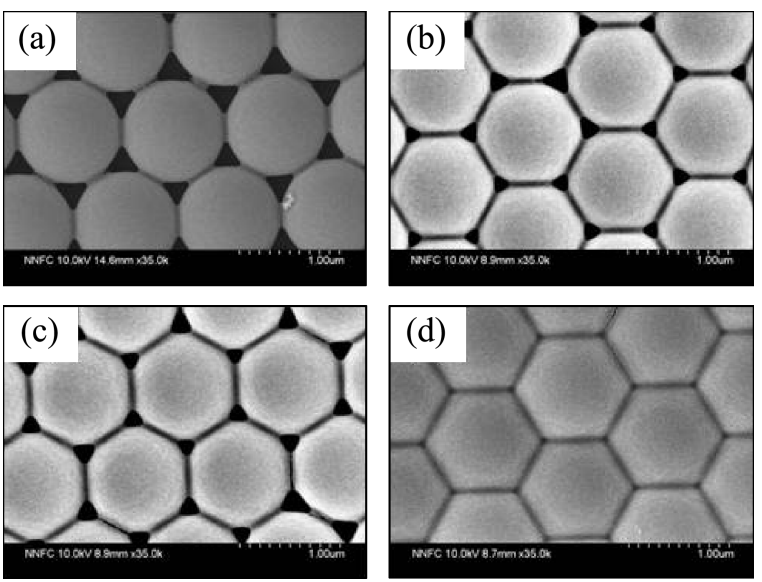

Figure 1. SEM images of PS particles in pure $\mathrm{CO}_{2}$ at $40{ }^{\circ} \mathrm{C}$ and 80 bar for variable time conditions: (a) 30 minutes, (b) 1 hour, (c) 2 hours, (d) 5 hours.

induce the clogging of pores around the PS particles, prohibiting the coating of silica onto the PS templates. Thus, it is necessary to determine the optimal pressure and temperature ranges in which to conduct the silica coating in $\mathrm{scCO}_{2}$.

The effect of $\mathrm{CO}_{2}$ was determined by exposing PS samples $(1.1 \mu \mathrm{m})$ to $\mathrm{scCO}_{2}$ at various temperatures, pressures, and treatment times. The standard conditions were $40{ }^{\circ} \mathrm{C}, 80 \mathrm{bar}$, and $30 \mathrm{~min}$. Figure 1 shows four SEM images of the $1.1-\mu \mathrm{m}$ PS spheres exposed to $\mathrm{scCO}_{2}$ for four treatment times.

Figure 1(a) shows that the $\mathrm{CO}_{2}$ did not significantly affect the spherical shape of the PS particles under these conditions for a treatment time of $30 \mathrm{~min}$. However, the templates exposed to $\mathrm{scCO}_{2}$ for 1 to $5 \mathrm{hrs}$ lost their spherical particle structure and were gradually transformed to a hexagonal structure. The annealing effect caused the voids between the PS particles to become smaller, changing the behavior of the silica precursor, which can permeate into the interstices. This phenomenon is not desirable for our experimental purpose because it can obstruct the synthesis of inverse opals. Therefore, we set the reaction time below $30 \mathrm{~min}$.

Once the reaction time was set at $30 \mathrm{~min}$, the effects of
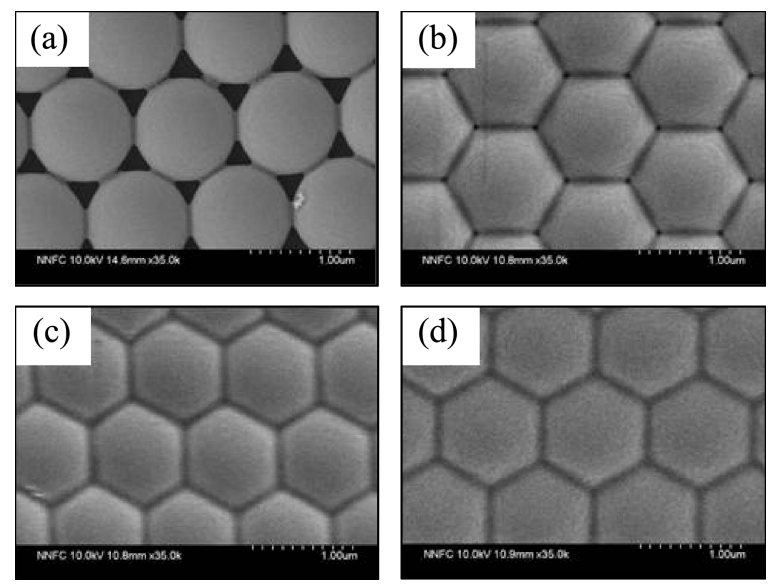

Figure 2. SEM images of PS particles in pure $\mathrm{CO}_{2}$ at 80 bar and 30 minutes for variable temperature conditions: (a) $40{ }^{\circ} \mathrm{C}$, (b) $50{ }^{\circ} \mathrm{C}$, (c) $60^{\circ} \mathrm{C}$, (d) $70^{\circ} \mathrm{C}$. 

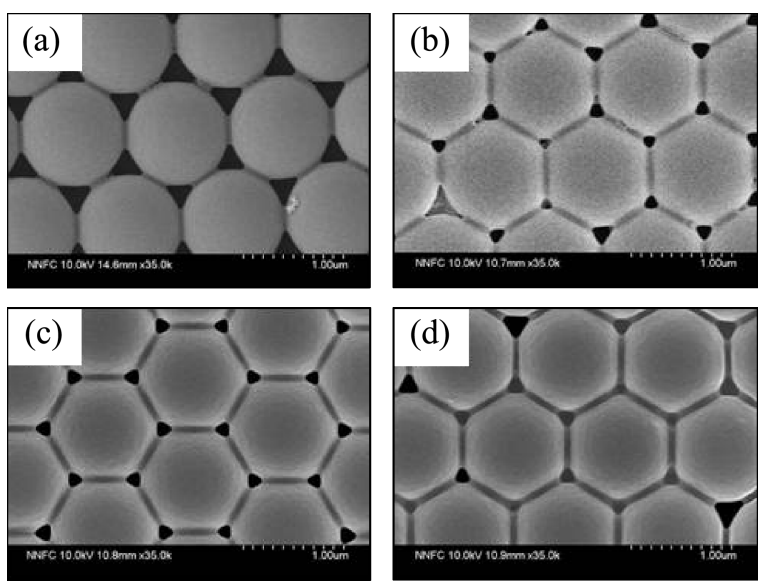

Figure 3. SEM images of PS particles in pure $\mathrm{CO}_{2}$ at $40{ }^{\circ} \mathrm{C}$ and 30 minutes for variable pressure conditions: (a) 80 bar, (b) 100 bar, (c) 120 bar, (d) 140 bar.

temperature and pressure were studied by varying the temperature from 40 to $70{ }^{\circ} \mathrm{C}$ and the pressure form 80 to 140 bar. As shown in Figure 2, PS particles transformed rapidly when the temperature was higher than $40{ }^{\circ} \mathrm{C}$. In addition, Figure 3 shows the SEM images of the templates with a variation in pressure. At pressures lower than 140 bar, at a temperature of $40^{\circ} \mathrm{C}$ and a reaction time of $30 \mathrm{~min}$, no rapid transformation in particle shape and pore size were observed. This indicates that the particle shape is transformed to a hexagonal form as the pressure is increased to 140 bar. These results show that the stability of the PS particle is more sensitive to temperature changes than to pressure changes.

Generally, the reaction rate for sol-gel deposition in $\mathrm{scCO}_{2}$ is accelerated with increase in temperature, and reduced with increase in pressure. When a polymer is treated with $\mathrm{scCO}_{2}$, compressed $\mathrm{CO}_{2}$ can infiltrate into the voids between the polymer molecules, leading to possible swelling of the polymer particles and decreased interactions between the functional groups of the polymer. ${ }^{17,18}$ This process makes the polymer more flexible, and reduces the $T_{g}$ which causes the transformation of the spherical structure of PS. ${ }^{19}$ Thus, in the production of $\mathrm{SiO}_{2}$ inverse opals, a temperature close to or greater than the $T_{g}$ of the PS particle causes the shape of the particles to change, a fact which is important if the temperature is increased to improve the reaction rate. The reaction rate decreases if the temperature is set near the critical temperature of $\mathrm{CO}_{2}$ and if the $\mathrm{CO}_{2}$ pressure is high. Therefore, for our purpose, it was optimal to set the pressure near the critical point of $\mathrm{CO}_{2}$ (80 bar).
Preparation of $\mathrm{SiO}_{2}$ Inverse Opals in $\mathbf{S c C O}_{2}$. Based on the results of the stability test, we determined the optimal condition for the coating of silica to be $40{ }^{\circ} \mathrm{C}$ and 80 bar. Before they were coated, the PS templates were treated with BSA, which is an acid catalyst for sol-gel reaction and enhance the reactions that enhances only the reaction occurring on the particle surface. Three vials containing the PS templates, DI water, and TEOS (a silica precursor) were placed in a reactor and the pressure was increased while the $\mathrm{CO}_{2}$ gas was purged into the reactor. Above the critical point of $\mathrm{CO}_{2}, \mathrm{scCO}_{2}$ dissolved the water and TEOS to form a homogenous solution. After the reaction, the pressure was lowered, and all of the $\mathrm{CO}_{2}$ was vented from the sample. The samples were then heat-treated at $500{ }^{\circ} \mathrm{C}$ to remove the polymeric templates, leaving behind silica inverse opals. Figure 4 is a schematic diagram of the process for making $\mathrm{SiO}_{2}$ inverse opals using $\mathrm{scCO}_{2}$.

SEM pictures of the resulting materials (Figure 5 and Figure 6) reveal that they are highly porous materials that have an inverted form relative to that of the templates.

Figure 5 shows the SEM images of the silica inverse opals produced with various reaction times in $\mathrm{scCO}_{2}$. During the stability test, we found that the polymeric templates retained its original form until it was exposed to $\mathrm{ScCO}_{2}$ for $30 \mathrm{~min}$; for this reason, we set the reaction time to be approximately $30 \mathrm{~min}$. After a reaction time of $20 \mathrm{~min}$, a thin shell of silica was observed; after a reaction time of $30 \mathrm{~min}$, the silica inverse opals were composed of thick, particulate silica. After reaction times of more than 30 min (data not shown), we found that the silica was over-coated on the PS opals and no inverse opals were obtained after the coating process.

We have also applied poly(styrene-co-methacrylic acid) (PS-MA) particles and their assembled opal structures as a template. In this case, because of the acidic groups impregnated in the PS particles, BSA-treatment of the surface of the particles is not required.

Figure 6 illustrates the SEM images of the inverse opals
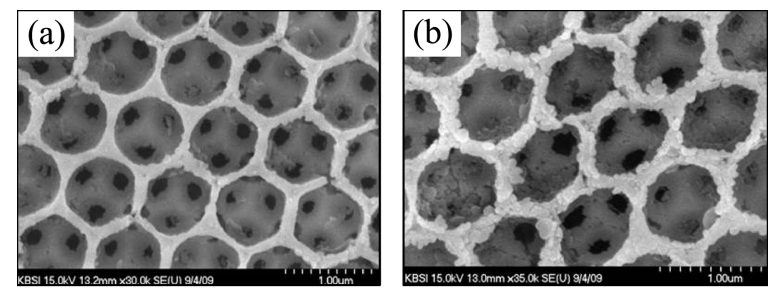

Figure 5. $\mathrm{SEM}$ images of obtained $\mathrm{SiO}_{2}$ inverse opals using PS particles as a template at $40{ }^{\circ} \mathrm{C}$ and 80 bar for variable time conditions: (a) 20 minutes, (b) 30 minutes.

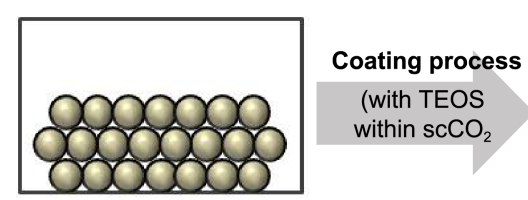

PS opal template

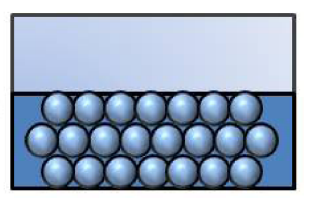

Coated with silica precursor

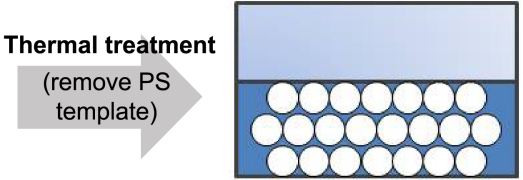

Inverted structure of $\mathrm{SiO}_{2}$

Figure 4. A schematic diagram of the process for obtaining $\mathrm{SiO}_{2}$ inverse opals within $\mathrm{scCO}_{2}$. 

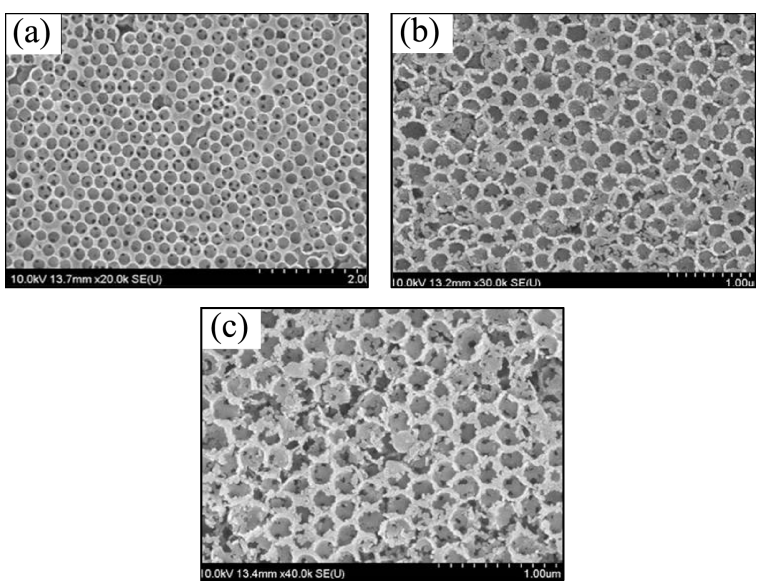

Figure 6. SEM images of obtained $\mathrm{SiO}_{2}$ inverse opals using 235 nm PS-MA particles as a template at $40{ }^{\circ} \mathrm{C}$ for variables time and pressure conditions: (a) 30 minutes, 80 bar (b) 40 minutes, 80 bar (c) 30 minutes, 100 bar.

that were created using PS-MA templates with a particle size of $235 \mathrm{~nm}$. The inverse opals that were created with a reaction time of $40 \mathrm{~min}$ were over-coated with the silica precursor compared to those created with a reaction time of 30 $\min$. After a reaction time of $1 \mathrm{hr}$, the walls of the inverse opals were excessively over-coated. The effect of the $\mathrm{CO}_{2}$ pressure on the condition of the coating can be observed by comparing Figure 6(a) and Figure 6(c). These figures show that the silica coating was thicker when the pressure was 100 bar than when it was 80 bar. At a pressure greater than 100 bar, it is expected that the cavities in the particles might be destroyed, and therefore, the process would not replicate the structure of the template.

In addition to being a rapid reaction process, another advantage of using the supercritical deposition method to synthesize inverse opals is a reduction in the shrinkage during the coating process. It has been reported ${ }^{20,21}$ that the shrinkage in conventional liquid processes was over 20 to $30 \%$. As we calculated from the SEM images, after reaction in $\mathrm{scCO}_{2}$ and calcination, the silica-coated particles, which are fabricated from a polymeric latex molecule with a size of $1.1 \mu \mathrm{m}$, have a size of approximatley $830 \mathrm{~nm}$ (Figure 5(a)). This change represents a shrinkage of approximately $17 \%$ with respect to the original molecule size. With another template which was composed of 235-nm PS-MA molecules, the pore diameter of the inverted material was close to 200 $\mathrm{nm}$ (Figure 6(a)). Compared to the original template particle, the pore size of PS-MA inverse opals shrank by $15 \%$. From these results, it is clear that supercritical deposition can reduce the shrinkage effect compared to the conventional liquid process.

\section{Summary}

We have fabricated $\mathrm{SiO}_{2}$ inverse opals by sol-gel deposition of silica in $\mathrm{scCO}_{2}$ on PS templates. In this process, opal structures of PS particles treated by organic acids were used as a template for the silica coating and the $\mathrm{scCO}_{2}$ was used as a medium for the sol-gel reaction of the silica precursor, TEOS. Silica inverse opals were obtained after coating and subsequent calcinations. In this study, the effects of temperature, pressure, and reaction time on the stability of PS opal templates were investigated. As the reaction time was increased at a pressure of $80 \mathrm{bar}$ and a temperature of $40^{\circ} \mathrm{C}$, the PS opal templates showed a melting and subsequent necking between particles after $1 \mathrm{hr}$. Annealing of PS opal templates occurred at temperatures above $50{ }^{\circ} \mathrm{C}$ (at a pressure of $80 \mathrm{bar}$ ) or a pressures of $100 \mathrm{bar}$ (at a temperature of $40{ }^{\circ} \mathrm{C}$ ). Thus, the coating of silica under $\mathrm{scCO}_{2}$ was performed at a fixed temperature of $40{ }^{\circ} \mathrm{C}$ and a pressure ranging from 80 to 100 bar. Temperatures and pressures greater than these destroyed the cavities among the particles, and therefore, $\mathrm{SiO}_{2}$ inverse opals were not obtained. After the optimal conditions were determined, we applied the silica coating in $\mathrm{scCO}_{2}$ on PS templates, using these conditions and controlling the time of the sol-gel reaction. As the reaction time increase, the thickness of the silica coating increased; however, after a reaction of more than $40 \mathrm{~min}$, the excessive coating fully covered the PS opal templates and no macropores were formed.

Compared to previously described mechanisms of coating in the liquid or gas phase, sol-gel coating in $\mathrm{scCO}_{2}$ possesses advantages in that no reactants and byproducts remain on the template samples, and selective coating on the surface of the template can be achieved. Moreover, a simple change in the reaction temperature and/or the pressure of the $\mathrm{scCO}_{2}$ can change the solubility of the reaction medium, which might be useful to control the sol-gel reaction. We are currently investigating the microstructure of the coated silica shell in details and investigating the synthesis of $\mathrm{TiO}_{2}$ inverse opals, which might be useful for applications in photonic crystals because of their larger refractive index.

Acknowledgments. This work was supported by the National Research Foundation of Korea Grant funded by the Korean Government MEST, Basic Research Promotion Fund. This work was also supported by the Special Research Grant of Sogang University (2009).

\section{References}

1. Xia, Y.; Gates, B.; Yin, Y.; Lu, Y. Adv. Mater. 2000, 12, 693.

2. Moon, J. H.; Yang, S. M. Chem. Rev. 2010, 110, 547.

3. Piret, F.; Kwon, Y. U.; Su, B. L. Chem. Phys. Lett. 2009, 472, 207.

4. Biswas, R.; Sigalas, M. M.; Subramania, G.; Soukoulis, C. M.; Ho, K. M. Phys. Rev. B 2000, 61, 4549.

5. Bratton, D.; Yang, D.; Dai, J. Y.; Christopher, K. Polym. Adv. Techol. 2006, 17, 94.

6. Wang, X.; Xu, J. F.; Su, H. M.; Zeng, Z. H.; Chen, Y. L.; Wang, H. Z. Appl. Phys. Lett. 2003, 82, 2212.

7. Sanders, J. V. Nature 1964, 204, 1151.

8. Stein, A.; Schroden, R. Curr. Opin. Solid State Mat. Sci. 2001, 5 , 553.

9. Lopez, C. Adv. Mater. 2003, 15, 1679.

10. McHugh, M. A.; Krukonis, V. J. Supercritical Fluid Extraction: Principles and Practice; Butterworths: Boston, 1986.

11. Bae, Y. C.; Gulari, E. J. Appl. Polym. Sci. 1997, 63, 459.

12. Moon, J. H.; Cho, Y. S.; Yang, S. M. Bull. Korean Chem. Soc. 
2009, 30, 2245.

13. Cabanas, A.; Enciso, E.; Carbajo, M. C.; Torralvo, M. J.; Pando, C.; Renuncio, J. A. R. Chem. Mat. 2005, 17, 6137.

14. Tomasko, D. L.; Li, H.; Liu, D.; Han, X.; Wingert, M. J.; Lee, J. L.; Koelling, K. W. Ind. Eng. Chem. Res. 2003, 42, 6431.

15. Condo, P. D.; Johnston, K. P. Macromolecules 1992, 25, 6119.

16. Chiou, J. S.; Barlow, J. W.; Paul, D. R. J. Appl. Polym. Sci. 1985, 30, 2633.

17. Cabanas, A.; Enciso, E.; Carbajo, M. C.; Torralvo, M. J.; Pando,
C. Langmuir 2006, 22, 8966.

18. Wissinger, R. G.; Paulatis, M. E. J. Polym. Sci., Pt. B-Polym. Phys. 1991, 29, 631 .

19. Abramowitz, H.; Shah, P. S.; Green, P. F.; Johnston, K. P. Macromolecules 2004, 37, 7316.

20. Holland, B. T.; Blanford, C. F.; Do, T.; Stein, A. Chem. Mat. 1999, $11,795$.

21. Carbajo, M. C.; Lopez, C.; Gomez, A.; Enciso, E.; Torralvo, M. J. J. Mater. Chem. 2003, 13, 2311. 